Report Number: LBNL-51491

\title{
SEARCH FOR CP VIOLATION IN HYPERON DECAYS
}

\author{
Piotr Zyla \\ Lawrence Berkeley National Laboratory \\ Berkeley, CA 94720, U.S.A.
}

Representing the Fermilab HyperCP collaboration

\section{DISCLAIMER}

This document was prepared as an account of work sponsored by the United States Government. While this document is believed to contain correct information, neither the United States Government nor any agency thereof, nor The Regents of the University of California, nor any of their employees, makes any warranty, express or implied, or assumes any legal responsibility for the accuracy, completeness, or usefulness of any information, apparatus, product, or process disclosed, or represents that its use would not infringe privately owned rights. Reference herein to any specific commercial product, process, or service by its trade name, trademark, manufacturer, or otherwise, does not necessarily constitute or imply its endorsement, recommendation, or favoring by the United States Government or any agency thereof, or The Regents of the University of California. The views and opinions of authors expressed herein do not necessarily state or reflect those of the United States Government or any agency thereof, or The Regents of the University of California. Ernest Orlando Lawrence Berkeley National Laboratory is an equal opportunity employer. 



\section{Search for CP Violation in Hyperon Decays}

Piotr Zyla ${ }^{\text {a* }}$

${ }^{\text {a} L a w r e n c e ~ B e r k e l e y ~ N a t i o n a l ~ L a b o r a t o r y, ~ B l d g . ~ 50 R-6040, ~ B e r k e l e y, ~ C A ~ 94720-8165 ~}$

Direct CP violation in nonleptonic hyperon decays can be established by comparing the decays of hyperons and anti-hyperons. For $\Xi$ decay to $\Lambda \pi$ followed by $\Lambda$ to $p \pi$, the proton distribution in the rest frame of Lambda is governed by the product of the decay parameters $\alpha_{\Xi} \alpha_{\Lambda}$. The asymmetry $A_{\Xi \Lambda}$, proportional to the difference of $\alpha_{\Xi} \alpha_{\Lambda}$ of the hyperon and anti-hyperon decays, vanishes if CP is conserved. We report on an analysis of a fraction of 1997 and 1999 data collected by the HyperCP (E871) collaboration during the fixed-target runs at Fermilab. The preliminary measurement of the asymmetry is $A_{\Xi \Lambda}=[-7 \pm 12($ stat $) \pm 6.2($ sys $)] \times 10^{-4}$, an order of magnitude better than the present limit.

\section{INTRODUCTION}

The phenomenon of $\mathrm{CP}$ violation is believed to be one of the necessary conditions for the creation of asymmetry between matter and anti-matter in the observed Universe [1]. The Standard Model predictions are insufficient to quantitatively describe the observation. It is thus important to study CP violation in a variety of systems. CP violation has been observed in the decays of neutral $\mathrm{K}$ and $\mathrm{B}$ systems. The HyperCP experiment was designed to probe direct CP symmetry in a sequence of hyperon decays: $\Xi \rightarrow \Lambda \pi, \Lambda \rightarrow p \pi$. This is a complimentary measurement to $\epsilon^{\prime} / \epsilon$ in the neutral $\mathrm{K}$ system, the only observation of direct $\mathrm{CP}$ violation.

\section{PHENOMENOLOGY OF NONLEP- TONIC HYPERON DECAYS}

The nonleptonic weak decay of $\Lambda$ to $p \pi$ is parity violating. The total angular momentum of the

\footnotetext{
* Representing the HyperCP collaboration: A. Chan, Y.C. Chen, C. Ho, P.K. Teng (Academia Sinica); W.S. Choong, G. Gidal, Y. Fu, P. Gu, T. Jones, K.B. Luk, B. Turko, P. Zyla (UC Berkeley and LBNL); C. James, J. Volk (FNAL); J. Felix (Guanajuato); R.A. Burnstein, A. Chakrovorty, D.M. Kaplan, L.M. Lederman, W. Luebke, D. Rajaram, H.A. Rubin, N. Solomey, Y. Torun, C.G. White, S.L. White (IIT, Chicago); N. Leros, J.P. Perroud (Lausanne); H.R. Gustafson, M.J. Longo, F. Lopez, H.K. Park (Michigan); K. Clark, M. Jenkins (S. Alabama); E.C. Dukes, C. Durandet, T. Holmstrom, M. Huang, L. Lu, K.S. Nelson (Virginia).
}

initial state is given by the spin $1 / 2$ of the $\Lambda$. In the final state we have a $p$ with spin $1 / 2$, a $\pi$ with spin 0 and the $p \pi$ orbital angular momentum. The conservation of angular momentum allows for parity-violating $S$ wave and parity-conserving $P$ wave of the final state orbital angular momentum to contribute to the decay amplitude. Assume that the $\Lambda$ 's are polarized and $\mathbf{P}_{\Lambda}$ is the polarization vector, the distribution of protons in the $\Lambda$ rest frame is linear in the cosine of the angle $\theta$ between the direction of proton momentum and $\mathbf{P}_{\Lambda}$. The slope is given by the product of magnitude of polarization and the $\alpha_{\Lambda}$ decay parameter:

$\frac{d N_{p}}{d \cos (\theta)}=\frac{N_{0}}{2}\left(1+\alpha_{\Lambda} \mathrm{P}_{\Lambda} \cos (\theta)\right)$.

The decay parameter $\alpha_{\Lambda}$ is proportional to the real part of the interference between the $S$ and $P$ waves of the final state:

$\alpha_{\Lambda}=\frac{2 R e\left(S^{*} P\right)}{|S|^{2}+|P|^{2}}$,

and so is a measure of the parity violation in the decay. The unpolarized $\Xi$ 's produce $\Lambda$ 's with longitudinal polarization $\mathrm{P}_{\Xi}$ given by the $\alpha_{\Xi}$ decay parameter, $\mathrm{P}_{\Xi}=\alpha_{\Xi}$. The slope of the proton distribution in the $\Lambda$ 's helicity frame is then given by the product $\alpha_{\Lambda} \alpha_{\Xi}$ of the decay parameters,

$\frac{d N_{p}}{d \cos (\theta)}=\frac{N_{0}}{2}\left(1+\alpha_{\Lambda} \alpha_{\Xi} \cos (\theta)\right)$. 
The CP operation transforms the $\Xi^{-} \rightarrow \Lambda^{0} \pi^{-}$ and $\Lambda^{0} \rightarrow p \pi^{-}$decays to the CP-conjugate decays, $\bar{\Xi}^{+} \rightarrow \bar{\Lambda}^{0} \pi^{+}$and $\bar{\Lambda}^{0} \rightarrow \bar{p} \pi^{+}$. It transforms the decay parameters $\alpha_{\Lambda}$ to $-\alpha_{\bar{\Lambda}}$ and $\alpha_{\Xi}$ to $-\alpha_{\bar{\Xi}}$. One can form the asymmetries,

$A_{\Lambda}=\frac{\alpha_{\Lambda}+\alpha_{\bar{\Lambda}}}{\alpha_{\Lambda}-\alpha_{\bar{\Lambda}}}, \quad A_{\Xi}=\frac{\alpha_{\Xi}+\alpha_{\bar{\Xi}}}{\alpha_{\Xi}-\alpha_{\Xi}}$,

and

$A_{\Xi \Lambda}=\frac{\alpha_{\Xi} \alpha_{\Lambda}-\alpha_{\bar{\Xi}} \alpha_{\bar{\Lambda}}}{\alpha_{\Xi} \alpha_{\Lambda}+\alpha_{\bar{\Xi}} \alpha_{\bar{\Lambda}}} \approx A_{\Xi}+A_{\Lambda}$,

that measure direct $\mathrm{CP}$ violation. They are equal to zero for $\mathrm{CP}$ conserving decays.

\section{THEORETICAL EXPECTATIONS AND EXPERIMENTAL RESULTS}

The theoretical predictions for $A_{\Xi}, A_{\Lambda}$ are summarized in the Ref. [2]. Calculations in the framework of the Standard Model lead to small asymmetries, $\sim 10^{-5}$ level, and give only order of magnitude estimates. The measurements of direct $\mathrm{CP}$ violation in hyperon decays are complementary to $\epsilon^{\prime} / \epsilon$ measurements of direct $\mathrm{CP}$ violation in the neutral $K$ system. While CP asymmetries $A_{\Lambda}$ and $A_{\Xi}$ are sensitive to both parity-violating and parity-conserving final state contributions the $\epsilon^{\prime} / \epsilon$ is sensitive only to parityviolating part. That raises possibility of large SUSY contributions to $A_{\Lambda}$ up to $10^{-3}$ [3] while preserving the measured value of $\epsilon^{\prime} / \epsilon$.

The most precise experimental measurements of hyperon asymmetries were performed by the PS185 collaboration at LEAR [4], $A_{\Lambda}=-0.013 \pm$ 0.022 , and E756 at FNAL [5], $A_{\Xi \Lambda}=0.012 \pm$ 0.014 . The results are consistent with no observation of $\mathrm{CP}$ violation with the best precision at the $10^{-2}$ level.

\section{THE HYPERCP EXPERIMENT}

The HyperCP, FNAL E871, experiment was designed to probe $A_{\Xi \Lambda}$ at the $10^{-4}$ level in the decay sequence $\Xi \rightarrow \Lambda \pi, \Lambda \rightarrow p \pi$. It collected data with a simple and fast spectrometer, Figure 1. The $800 \mathrm{GeV}$ proton beam scattered off a copper target. A chunk of a secondary beam
Figure 1. Schematic of the HyperCP spectrometer.

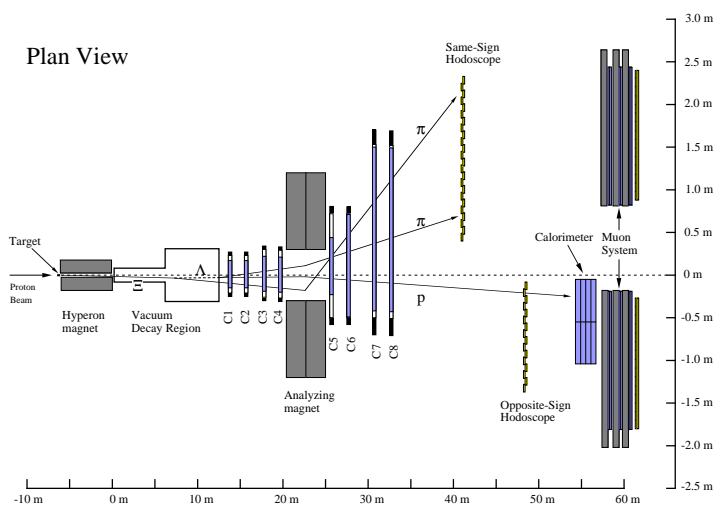

particles was selected by a 6 -meter long collimator embedded in a 1.67 Tesla dipole magnet. The charged particles were bent about 19.5 mRadians upwards and had momenta between 120 and 250 $\mathrm{GeV} / \mathrm{c}$ at the collimator exit. They next entered a 13-meters long vacuum pipe. Charged decay products were tracked by four Multiwire Proportional Chambers (MWPC), four planes each, with a distance between wires from 1 to $1.25 \mathrm{~mm}$. The 4-meter long dipole magnet with the $1.4 \mathrm{GeV} / \mathrm{c}$ momentum kick in the horizontal direction was used to measure particle momenta. It was followed by another set of four MWPCs, four planes each, with a wire distance from 1.5 to $2 \mathrm{~mm}$. The main trigger consisted of two hodoscopes, one for particles with positive and one with negative charge, and a hadronic calorimeter. The trigger required one hit per hodoscope in coincidence with a minimum energy deposition in the calorimeter. The calorimeter energy requirement suppressed particles coming from secondary interactions with spectrometer material and muons. The trigger elements were followed by two muon identification systems, not used in this analysis. To collect data with a secondary beam of opposite charge we changed the direction of the magnetic field in both the collimator and momentum analyzing magnets. That kept the geometry of 
the spectrometer CP invariant. We also changed the length of the target to assure similar rates of secondary beam in the spectrometer for both charge polarities of the beam. The polarities were changed every several hours during the run to minimize the effect of slow systematic changes in the spectrometer.

The HyperCP collected data during the 1997 and 1999 fixed target runs at FNAL. The fully reconstructed $\sim 2.5 \times 10^{9} \Xi^{-}$and $\bar{\Xi}^{+}$decays allow for a measurement of $A_{\Xi \Lambda}$ with $\sim 2 \times 10^{-4}$ statistical precision.

\section{ANALYSIS AND RESULTS}

The positive and negative secondary beams of $\Xi$ 's were both produced by protons interacting with the copper target. The momentum spectra at the exit of the collimator are slightly different, leading to a difference in geometric acceptance. That would give rise to an asymmetry if the acceptance is not corrected for. We have developed two techniques to address this effect: a hybrid Monte Carlo (HMC) method [6] and a weighting method.

The HMC method is a Monte Carlo that preserves the maximum possible information from real events. For each real input event $10 \mathrm{HMC}$ events are created. They use all the measured quantities of the input event except for the proton angular distribution in the $\Lambda$ helicity frame: the $\mathrm{HMC} \cos (\theta)$ is generated uniformly. Every HMC event is subject to codes simulating multiple scattering, earth magnetic field, digitization, detector and trigger with measured efficiencies and the same reconstruction, apertures, and selection process as the input event. The $\cos (\theta)$ distribution of the accepted HMC events is adjusted with the weights that depend on the value of $\alpha_{\Xi} \alpha_{\Lambda}$ treated as a parameter to fit the $\cos (\theta)$ distribution of the real input events. This procedure relies heavily on the reconstructed information of the real event, e.g. the momentum of the $\Xi$ at the exit of the collimator, and naturally takes into account the production difference between the $\Xi^{-}$and $\bar{\Xi}^{+}$ beams. The HMC method was tested with Monte Carlo generated sample with the PDG value of $\alpha_{\Xi} \alpha_{\Lambda}=-0.2927( \pm 0.0070)$ [7]. The obtained re- sult, $-0.2953 \pm 0.0029$, was consistent with the input.

We have analyzed, using the HMC method, a sample of randomly selected $\Xi$ decays from the full data set. It consisted of about $15 \times 10^{6} \Xi^{-}$ and $30 \times 10^{6} \Xi^{+}$input events. The measured value of $\alpha_{\Xi} \alpha_{\Lambda}$ as a function of run number is shown in Figure 2. The runs below 3600 are the 1997 data

Figure 2. A measured value of $\alpha_{\Xi} \alpha_{\Lambda}$ as a function of run number.

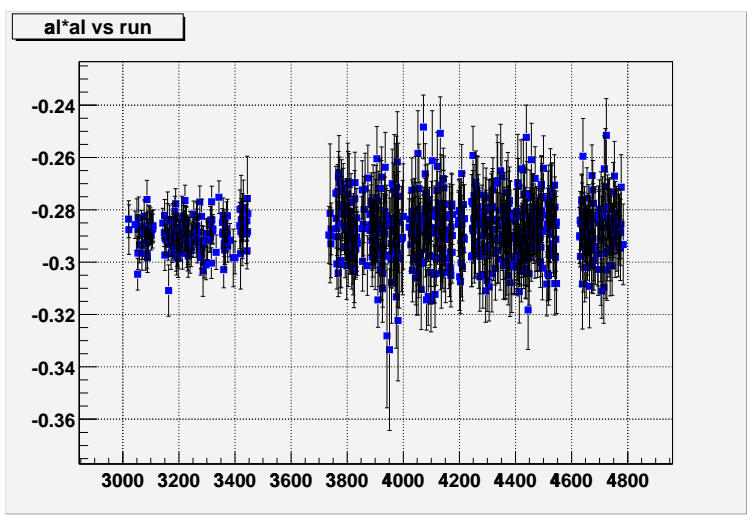

and above 3600 are the 1999 data samples. The error bars are statistical only. The best fit value to all data is $\alpha_{\Xi} \alpha_{\Lambda}=-0.2880 \pm 0.0004$ (stat), with $\chi^{2}$ of 26 per 19 degrees of freedom, and is consistent with the PDG average. Figure 3 shows the $\cos (\theta)$ distributions of the HMC events before and after weighting. The comparison of real events and the HMC distributions is shown in the insert.

The potential sources of the bias of the asymmetry measurement are the differences between the running conditions between positive and negative polarities of the beam of $\Xi_{\mathrm{s}}$. Listed in Table 1 are the differences in: beam polarization, the beam rate in the spectrometer, the backgrounds included in the analyzed sample and the shape of momentum dependent interaction between $p\left(\pi^{-}\right)$and $\bar{p}\left(\pi^{+}\right)$with the material of the 
Figure 3. HMC $\cos (\theta)$ distributions. Solid line shows the accepted HMC events. Dashed line is the same distribution after weighting with the best fit value of $\alpha_{\Xi} \alpha_{\Lambda}$. The insert shows the ratio of input to $\mathrm{HMC} \cos (\theta)$ distributions.

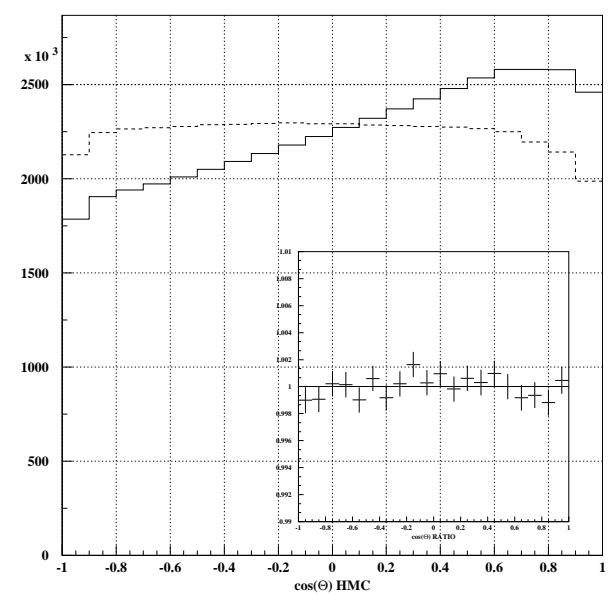

spectrometer. The uncertainties in the measurement of the efficiencies of the wire chambers and trigger elements were propagated to the uncertainty of the asymmetry. The total systematic uncertainty was obtained by combining the listed uncertainties in quadrature.

Based on the measured values of $\alpha_{\Xi} \alpha_{\Lambda}$ and $\alpha_{\bar{\Xi}} \alpha_{\bar{\Lambda}}$ with the HMC method we obtained a preliminary $\mathrm{CP}$ asymmetry

$A_{\Xi \Lambda}=[-7 \pm 12($ stat $) \pm 6.2($ sys $)] \times 10^{-4}$.

It is consistent with no $\mathrm{CP}$ violation.

Another way to correct for the difference in the acceptance of $\Xi^{-}$and $\bar{\Xi}^{+}$hyperon beams is to weight the events to match the minimal set of kinematic and geometric characteristics of the beam. It was verified with $\mathrm{MC}$ samples that the method removes the bias due to production differences while preserving the input $\mathrm{CP}$ asymmetry of the MC data. The analysis with the weighting method is in progress.
Table 1

Summary of systematic uncertainties of the measured value of $A_{\Xi \Lambda}$.

\begin{tabular}{lc}
\hline Study & $\delta A_{\Xi \Lambda}\left(10^{-4}\right)$ \\
\hline Polarization & 0.4 \\
Rate dependence & 3.4 \\
Background & 2.4 \\
Hodo. Eff. & 1.7 \\
Cal. Eff. & 1.8 \\
PWC Eff. & 2.7 \\
B Field & 2.2 \\
Interaction diff. & 0.5 \\
\hline Total & 6.2 \\
\hline
\end{tabular}

\section{SUMMARY}

Based on a representative sample of the full HyperCP data set we measured a preliminary $\mathrm{CP}$ asymmetry,

$$
A_{\Xi \Lambda}=[-7 \pm 12(\text { stat }) \pm 6.2(\text { sys })] \times 10^{-4} .
$$

The value of asymmetry is consistent with zero at the $10^{-3}$ level. The total systematic uncertainty is limited by the sample statistics, and is expected to reduce for the full data set.

\section{REFERENCES}

1. A.D. Sakharov, ZhETF Pis'ma 5, 32 (1967).

2. S. Pakvasa, hep-ph/0002210

3. X.G. He, H. Murayama, S. Pakvasa, G. Valencia, Phys. Rev. D61 (2000) 71701.

4. P.D. Barnes et al., Phys. Rev. C54 (1996) 1877.

5. K.B. Luk et al., Phys. Rev. Lett. 85 (2000) 4860-4863.

6. G. Bunce, NIM 172 (1980), 553-557.

7. D.E. Groom et al., Eur. Phys. J. C15 (2000), 1-878. 